\title{
INVESTIGATION THE EFFECT OF ROUGHNESS CONTROL COEFFICIENT IN GROWTH OF CRYSTAL MATERIALS BY NANO EPITAXIAL UNDER A ONE-DIMENSIONAL BOUNDED DOMAIN
}

\author{
S.Azizi ${ }^{1,2}$, N. Maleki-Jirsaraei ${ }^{3}$, A.Yagi $^{4}$ \\ ${ }^{1,3}$ Complex Systems Lab., Physics Department, Al-Zahra University, Tehran, 199389117 Iran \\ 2,4Department of Physics, Osaka University, 1-1 Yamadaoka, Suita, Osaka 565-0871, Japan \\ s.azizi@alzahra.ac.ir, maleki@alzahra.ac.ir, A.yagi@ist.osaka-u.ac.jp
}

\begin{abstract}
This paper continues a study on the initial-boundary value problem for a semilinear parabolic equation of fourth order which has been presented by Johnson Orme-Hunt-GraffSudijono-Sauder-Orr [1] to describe the large-scale features of a growing crystal surface under molecular beam epitaxial. In the preceding papers [2, 3] we constructed a dynamical system generated by the problem and showed that every trajectory converges to some stationary solution at $t \rightarrow \infty$. In the subsequent paper [4] we succeeded in proving the stability of the model equation through an analytically under two-dimensional bounded domain. This paper is then devoted to investigating the stationary point of the roughness control coefficient for the same equation of preceding papers via numerical computations; in order to evaluation the effects of surface diffusion and roughening on stability and instability of null solutions. Also, the main resolution of this study is to manage these objectives under a one-dimensional domain with homogeneous Dirichlet boundary conditions. For this purpose, first we shall construct a discretization scheme by FiniteDifference Methods for the fourth order the equation and second we will investigate the some numerical results of a null stationary solution based on [4, theorem 3.1 and 3.2] to observe how changes the structure of stationary solutions with the changing the surface diffusion and roughening control coefficient in one-dimensional bounded domain. And finally, we shall demonstrate the instability of a null solution which is an inhomogeneous stationary solution.
\end{abstract}

Keywords: Molecular Beam Epitaxial, Fourth order equation, Numerical computations, Surface diffusion Roughening, Finite-Difference Methods, Zero Fixed solution or Null Stationary solution.

\section{Introduction}

The one of the most accomplished achievements of Molecular beam epitaxial (MBE) is the Nanostructures that permitted the formation of atomically flat and abrupt hetero-interfaces and is one of useful techniques that enable us to grow structures with very high precision in the vertical direction, such as monolayer-thin interfaces or atomically flat surfaces. But there still remains the major challenge of growing surfaces which are structured laterally. A theoretical contribution in order to control the unstable growth may then lie in understanding its basic mechanisms. In the present study, we relate to the problem of the initial boundary value for a nonparallel parabolic equation of the fourth order in

$$
\begin{cases}\frac{\partial u}{\partial t}=-a \Delta^{2} u-\mu \nabla \cdot\left[\frac{\nabla u}{1+|\nabla u|^{2}}\right] & \text { in } \Omega \times(0, \infty), \\ u=\frac{\partial u}{\partial n}=0 & \text { on } \partial \Omega \times(0, \infty), \\ u(x, 0)=u_{0}(x) & \text { in } \Omega,\end{cases}
$$

a domain of one-dimensional. We assume that $\Omega \subset R^{2}$, such a problem has been presented by Johonson- Orme [1], in order to describe the growth of the surface of the crystal material under MBE. Here, $u=u(x, t)$ is height of the surface at the direction $x$ and at timet. The idiom $-a \Delta^{2} u$ in the equation of (1.1) it indicates the emission of a surface due to the chemical potential difference that is proportional to the curvature of the surface. Therefore, atoms tend to migrate from large curvature to those of a small one. Simultaneously, the idiom $-\mu \nabla \cdot\left[\frac{\nabla u}{1+|\nabla u|^{2}}\right]$ indicates the effect of surface fragility, $\mu$ a positive constant called the surface 
hardness factor or surface roughening coefficient. Such a simulation arises from Schwebel's impediment. To solve the parabolic equation, it is naturally necessary that the boundary conditions be placed on the unknown function $u(x, t)$. In this study, we choose the boundary conditions of the homogeneous Dirichlet on $\partial \Omega$ In this sense, we assume that the height of the surface is always controlled at a constant level, $u=0$, along with the disappearance of natural derivatives, $\frac{\partial u}{\partial n}=0$. In the papers of Fujimura et al. [5, 6 and 7], Neumann's boundary conditions $\frac{\partial}{\partial n} \Delta u=0$ were studied. In the preceding paper [2] we created a dynamical system $\left(S(t), L_{2}(\Omega)\right.$ manufactured by $(1.1)$ for every initial mode $u_{0}(x, y)$, where $S(t)$ is a sequential nonlinear behaviour on $L_{2}(\Omega)$ specified by ecumenical solutions. And addition was shown that the dynamical system with having finite-dimensional attractor admits a In addition, it has been shown that the dynamic system adopts a Lyapunov function with finite absorption $\Phi(u)$ of the form (3.1) Their values are uniformly reduced. That is, a real evaluation function is defined for all state functions $u(x, y)$ its values are uniform for every path $t \rightarrow \infty$. Also in the paper [3] we presented that the situation $\bar{u}(x, y)$ is motionless if and only if $\bar{u}(x, y)$ is a critical value of the function, i.e. $\Phi(\bar{u})=0$, hence the long-term convergence of paths to some fixed solutions (1.1) depending on the initial functions. In [4], we managed to show the fixity and fluctuation of the zero solution analytically and numerically based on the two-dimensional boundary domain, and we also proved that a unique fixed solution is analogous.

This paper is a continuation of our study which uses numerical calculations of the critical point or fixed point of the roughness control coefficient for the problem of equation (1.1) in a finite domain of one dimension. We also show the fluctuation profiles of a zero solution in a finite domain based of force comparisons of surface diffusion parameter and roughness control coefficient.

In fact, we must prove that when the effects of surface penetration are stronger than the effects of roughness, then the zero solution is sustainable globally and in the meantime, when the effects of roughness are stronger than the effects of surface emission the zero solution is unstable. For this purpose, we first create an interpretive scheme with Finite-Difference Methods for equation (1.1), and secondly, we need to show some numerical results to observe how to change the structure of fixed solutions by changing the roughness control coefficient in a finite range of one-dimensional domain

\section{Methods}

\section{- Finite difference formulation for a one- dimensional problem}

FDMs are numerical methods for solving differential equations using their approximation to differential equations. In which the approximate differences are approximate derivatives and involves the mixing of the spatial domain, the differential equation, and boundary conditions. In this section of the FDMs, we use the equation of model (1.1) in the one-dimensional domain to diagnose the problem.

\section{A. Discretization of time and space in one- dimensional bounded domain}

The approximate solution in one dimensional case is obtained by $u\left(x_{i}, t_{n}\right) \approx u_{i}^{n}, \mathrm{i}=0 \ldots I$ and $n=0$, $1,2 \ldots$ The domain is divided in space and time and the approximate solution is calculated in space or time point, so the step size of the variable in the direction $x$ and the time step in $t$ are labelled with $\Delta x$ and $\Delta t$ respectively. First we make the time factor (1.1) .

$$
\frac{\partial u}{\partial t} \approx \frac{u\left(x_{i}, t_{n}\right)-u\left(x_{i}, t_{n-1}\right)}{\Delta t} \approx \frac{u_{i}^{n}-u_{i}^{n-1}}{\Delta t},
$$

The discrete space steps of the second derivative is obtained by the following way

$$
\begin{aligned}
\frac{\partial^{2} u}{\partial x^{2}} \approx & \frac{u\left(x_{i+1}, t_{n}\right)-2 u\left(x_{i}, t_{n}\right)+u\left(x_{i-1}, t_{n}\right)}{(\Delta x)^{2}} \\
& \approx D^{2} u_{i}^{n}=\frac{u_{i+1}^{n}-2 u_{i}^{n}+u_{i-1}^{n}}{(\Delta x)^{2}},
\end{aligned}
$$

Similarly, on the basis of these relations, for the fourth derivative we have the following approximation

$$
\frac{\partial^{4} u}{\partial x^{4}} \approx \frac{D^{2} u_{i+1}^{n}-2 D^{2} u_{i}^{n}+D^{2} u_{i-1}^{n}}{(\Delta x)^{2}}
$$

Also, discretization of $-\mu \cdot\left(\frac{\pi u}{1+\mid \sqrt{\Gamma u}]^{2}}\right)$ in Eq. (1.1) is presented in the following way

$$
D u_{i-\frac{1}{2}}^{n}=\frac{u_{i}^{n}-u_{i-1}^{n}}{\Delta x} . \quad D u_{i+\frac{1}{2}}^{n}=\frac{u_{i+1}^{n}-u_{i}^{n}}{\Delta x},
$$

Finally, the equation (1.1) after the division is as follows:

$$
u_{i}^{n+1}=u_{i}^{n}+\Delta t\left[\begin{array}{l}
-a \frac{D^{2} u_{i+1}^{n}-2 D^{2} u_{i}^{n}+D^{2} u_{i-1}^{n}}{(\Delta x)^{2}}- \\
\mu \frac{1}{\Delta x}\left(\frac{D u_{i+\frac{1}{2}}^{n}}{1+\left(D u_{i+\frac{1}{2}}^{n}\right)^{2}}-\frac{D u_{i-\frac{1}{2}}^{n}}{1+\left(D u_{i-\frac{1}{2}}^{n}\right)^{2}}\right)
\end{array}\right]
$$




\section{B. Discretization of boundary conditions in one- dimensional bounded domain}

The equation (1.1) is provided under the boundary conditions of homogeneous Dirichlet. Therefore, to calculate the boundary conditions onedimensional for $u=0$ we supposed

$$
u_{0}^{\mathrm{n}}=u_{I}^{n}=0 \text {, }
$$

where in $i=0, \ldots, I$ and $n=0,1,2$, on the other side for discrete the $\frac{\partial u}{\partial n}=0$, it is supposed that $u_{I+1}^{n}=u_{I-1}^{n}, u_{-1}^{n}=u_{1}^{n}$.

\section{Applications of MATLAB program in one- dimensional bounded domain}

The simulation process of equation (1.1) is presented by the MATLAB program. Expressed as follow

$$
\begin{aligned}
& u_{i}^{n+1}=u_{i}^{n}+\Delta t\left[- \text { a.part } 1-\mu \frac{1}{\Delta x}(\text { part } 2-\text { part } 3)\right] \\
& \operatorname{part~1:} \frac{D^{2} u_{i+1}^{n}-2 D^{2} u_{i}^{n}+D^{2} u_{i-1}^{n}}{(\Delta x)^{2}}= \\
& \frac{u_{i+1}^{n}-2 u_{i+1}^{n}+u_{i}^{n}}{\Delta x^{2}}-2 \frac{u_{i+1}^{n}-2 u_{i}^{n}+u_{i-1}^{n}}{\Delta x^{2}}+\frac{u_{i}^{n}-2 u_{i-1}^{n}+u_{i-2}^{n}}{\Delta x^{2}} \\
& \frac{u_{i+2}^{n}-4 u_{i+1}^{n}+6 u_{i}^{n}-4 u_{i-1}^{n}+u_{i-2}^{n}}{\Delta x^{4}} .
\end{aligned}
$$

part $2-$ part 3 :

$$
\begin{aligned}
& \frac{\frac{u_{i+1}^{n}-u_{i}^{n}}{\Delta x}}{1+\left(\frac{u_{i+1}^{n}-u_{i}^{n}}{\Delta x}\right)^{2}}-\frac{\frac{u_{i}^{n}-u_{i-1}^{n}}{\Delta x}}{1+\left(\frac{u_{i}^{n}-u_{i-1}^{n}}{\Delta x}\right)^{2}}= \\
& \frac{u_{i+1}^{n}-u_{i}^{n}}{\Delta x} \cdot \frac{(\Delta x)^{2}}{(\Delta x)^{2}+\left(u_{i+1}^{n}-u_{i}^{n}\right)^{2}}-\frac{u_{i}^{n}-u_{i-1}^{n}}{\Delta x} \cdot \frac{(\Delta x)^{2}}{(\Delta x)^{2}+\left(u_{i}^{n}-u_{i-1}^{n}\right)^{2}}= \\
& \frac{\Delta x\left(u_{i+1}^{n}-u_{i}^{n}\right)}{(\Delta x)^{2}+\left(u_{i+1}^{n}-u_{i}^{n}\right)^{2}}-\frac{\Delta x\left(u_{i}^{n}-u_{i-1}^{n}\right)}{(\Delta x)^{2}+\left(u_{i}^{n}-u_{i-1}^{n}\right)^{2}} .
\end{aligned}
$$

The definition of the Dirichlet boundary condition then is as follows:

$$
\begin{gathered}
\boldsymbol{u}_{\mathrm{O}}^{n}=\boldsymbol{u}_{I}^{n}=\mathrm{O}, \\
u_{I+1}^{n}=u_{I-1}^{n}, u_{-1}^{n}=u_{+1}^{n} .
\end{gathered}
$$

\section{Numerical Result}

In this section we illustrate some numerical examples of a zero fixed solution or in other words, null stationary solution based on force comparisons of surface diffusion parameter and roughness control coefficient to observe how changes the structure of fixed solutions. This claim is derived from the theorem [(3.1 and 3.2) and 4].

Proved in these sources that, (if $a d^{-2}>\mu_{x}$ then the zero solution is stable; otherwise, If $a d^{-2}<\mu_{\text {, }}$ then the null solution is unstable). In the our preceding paper [4] we shown that the stability and instability of the zero solution is determined by dominance in magnitude of the two coefficients a and $\mu$ to the other but with weight $d^{-2}$ for $a$. Such that these coefficients are the surface diffusion and roughening constantans; respectively. We also proved that if the Let $\Omega=\left(0, l_{1}\right)$, then weight constant $d$ is estimated by $d \leq \frac{\mathbb{l}_{1 l_{2}}}{\pi \sqrt{\mathbb{1}_{1}^{2}+i_{2}^{2}}}$. And according the If $\mu<\frac{\pi^{2}\left(i_{1}^{2}+i_{2}^{2}\right) a}{\pi_{1}^{2}+i_{2}^{2}}$, then the null solution is globally stable.

Then, we show the numerical results of a Fixed zero solution based on mentioned analytical results to observe how changes the structure of stationary solutions with the changing the surface diffusion and roughening control coefficient in one-dimensional bounded domain. And finally, we shall demonstrate the fixity and fluctuation of a zero solution which is an asymmetric constant solution. For this reason, we consider in (1.1) the distance of $\Omega=(0, l)$, where $l=1$. The coefficient $a$ is fixed as $a=1$ but $\mu>0$ it is considered as a control parameter. In this section constant $d$ is computed as $d \approx \frac{1}{\sqrt{2} \pi}$ (cf. [4, Theorem 5.4]). We also define the primary function as follows, $u_{0}(x)=0.1[\sin (2 \times 3.14 x)]_{s} \quad x \in \Omega_{x}$

Which is a disorder of the solution zero $u \equiv 0$. It is obvious that the solution of zero is a unique homogeneous fixed solution.

\section{A. Investigation of critical point or stationary point by roughening coefficient $\mu$ in one- dimensional}

The critical point of a function with real variables is a point in the domain of that function, where the function is not indeterminable at that point or its derivative is zero. 
In this section to find the static or fixed point we investigated the one dynamics system for several $\mu$ at the same time $t=150$. Then the critical point was observed about $\mu=13$. Note in the figure 4.1 that the remarkable point is that after a critical point any profiles with increased $\mu$ becomes asymmetric constant solution.
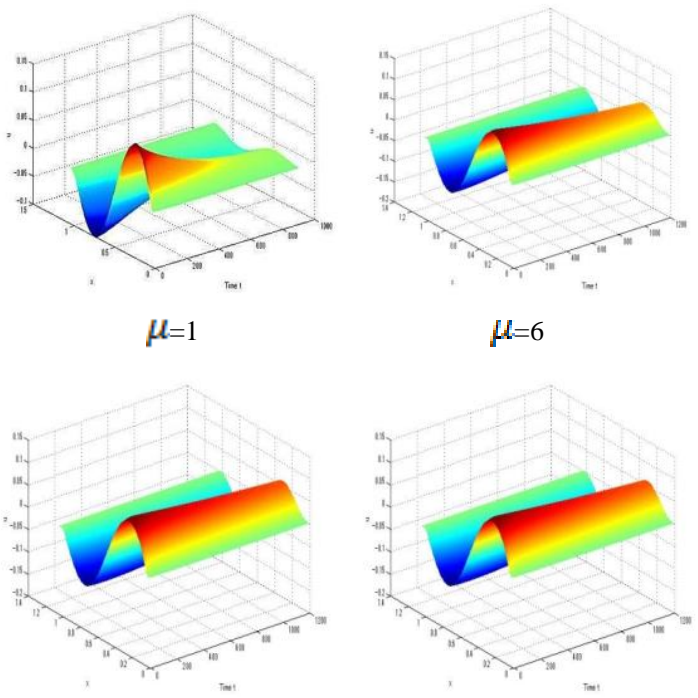

$\mu=12$

$\mu=13$
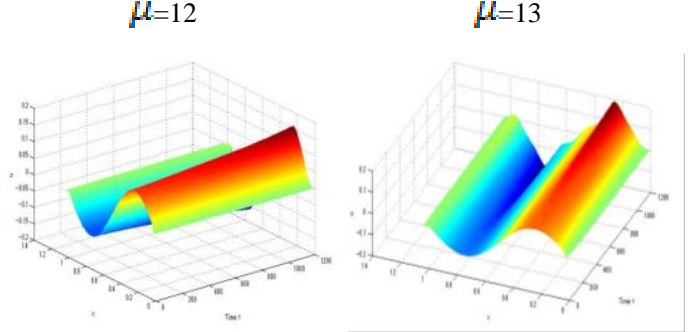

$\mu=15$

$\mu=20$
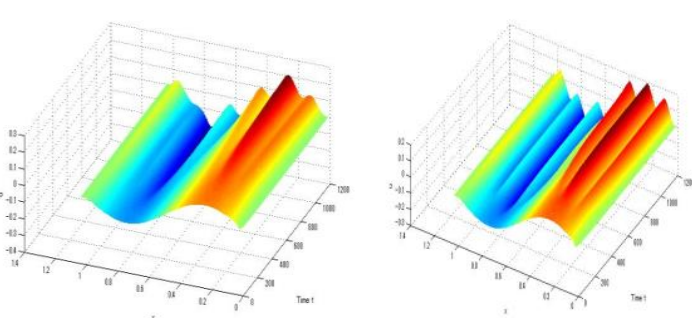

$\mu=40$

$\mu=60$

Figure 4.1: Dynamics for several $\mu$ at the same time $t=150$

\section{B. Investigation of stability and instability of null solution by controlling roughening coefficient in 1D}

We illustrate some numerical examples which show stability or instability of null solution by controlling roughening coefficient $\mu$. Set first $\mu=12_{s}$ such that $a d^{-2}>12_{x}$ then the null solution is stable. In this case, as seen by Figure 4.2, the solution tends to the null solution as $t \rightarrow \infty$. This means that the null stationary solution is stable, and the Lyapunov function along this trajectory is given by Figure 4.2(d). As explained above, after proving global existing of solutions, we construct a dynamical system and show that the dynamical system possesses a Lyapunov function, i.e., a real valued function whose values decreases monotonously along any trajectory. Furthermore, using the Lyapunov function, it is proved that every trajectory converges asymptotically to a stationary state which is a critical point of the Lyapunov function and in which the roughening effect is completely balanced with the surface diffusion.

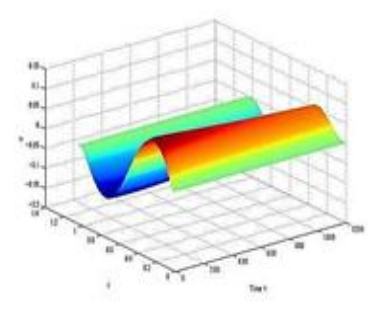

(a) $t=0$

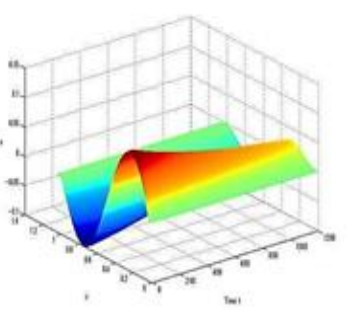

(c) $t=65$

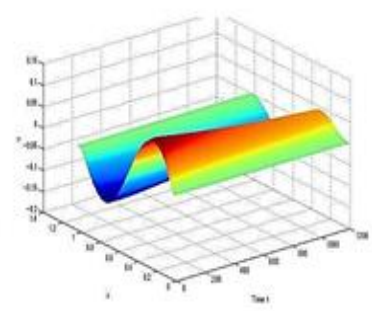

(b) $t=35$

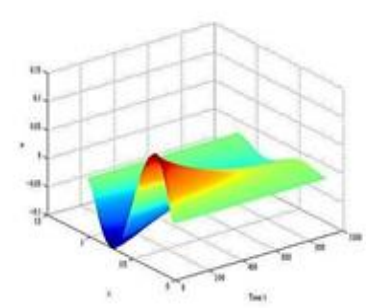

(d) $t=100$

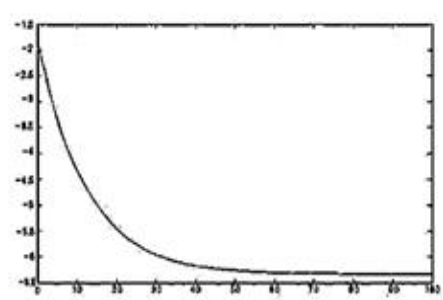

(d) Lyapunov function

Figure.4.2: Dynamics for $\mu=12$ in $1 D$

Take next $\mu=30$, such that $a d^{-2}<30$, then the zero solution is unstable or in other words there is a fluctuation on the surface. As seen by Figure 4.3, the solution no longer tends to the zero or null solution. Instead, with increasing time, the disruption of the two pillars of the pit (up, down) grows. This means that the fixed solution is unstable. The function diagram of the Lyapunov function along the path is shown in Figure 4.3 (j). 


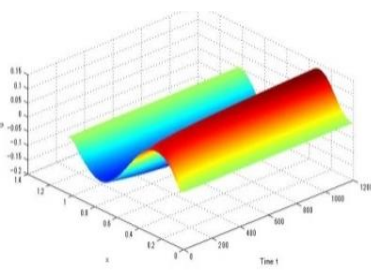

(a) $t=0$

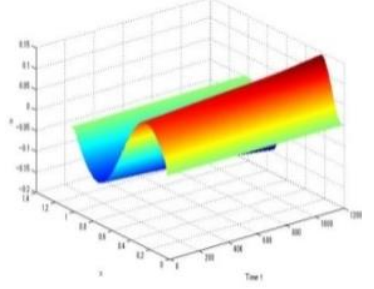

(a) $t=40$

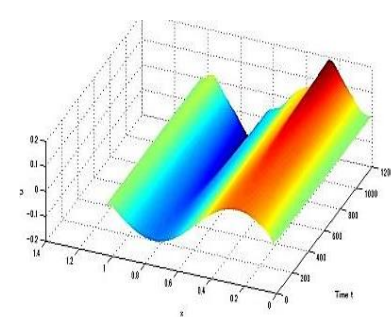

(a) $t=80$

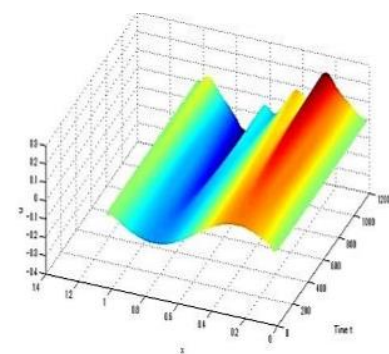

(a) $t=120$

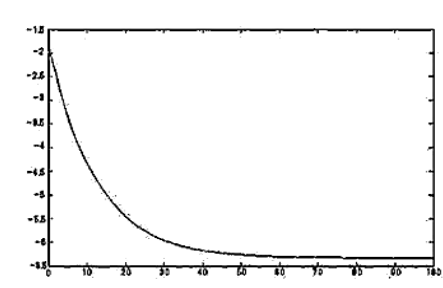

(j) Lyapunov function

Figure.4.3: Dynamics for $\mu=30$ in $1 D$

\section{Change of profiles by enhancement of roughening control coefficient}

In this section, we shall illustrate some numerical results to observe how change the profile of null stationary solution by enhancement of roughening coefficient. Therefore, in order to justify this section by numerical examples, we consider (1.1) with one of the roughening coefficient $\mu=13,25,60,90$, at the same time $t=200$. The coefficient $a$ is fixed as $a=1$. And the initial function and internal domain is same as previous section $\mathrm{A}$. When $\mu=13$ the solution tends to non-null stationary solution and as seen by Figure 4.4.a in each column there are 1 ridge. Set secondly $\mu=25$. In this case as before, the solution tends to non-null stationary solution and the perturbation grows into two columns of ridges, with the exception that the number of ridges in a column increases more than the case of $\mu=13$. In each column there are 2 ridges, as shown in Figure 4.4.b. Finally, set $\mu=60$ and 90 . As noted above, in these cases, the solutions tends to non-null stationary solution and the perturbation grows into two columns of ridges and the numbers of ridges in each column are more than those of other cases, as shown in Figure 4.4.c and 4.4.d.

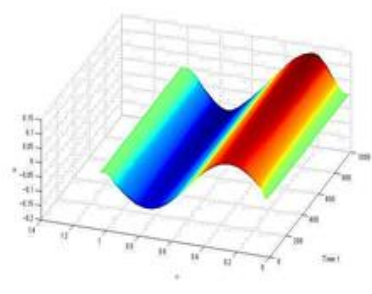

(a)

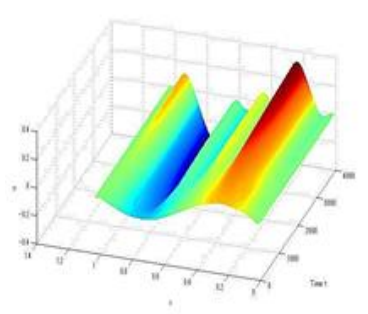

(c)

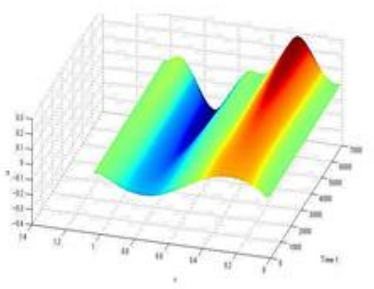

(b)

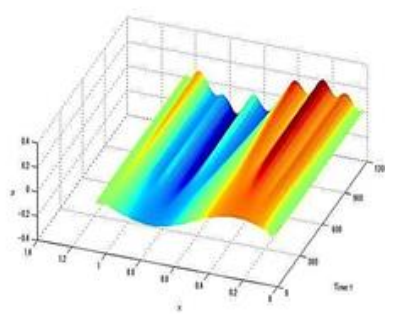

(d)
Figure.4.4: Dynamics for $\mu=13,25,60,90$ at the same time $t=200$

\section{Conclusions}

In this paper, we used the fourth order parabolic equation model containing two terms describing surface diffusion and roughening effect in order to describe the growing process of crystal surface by MBE. By equipping the homogeneous Dirichlet boundary conditions, we studied the model equation numerically in one dimensional bounded domain. 
We have investigated the stationary point of the roughness control coefficient and in order to obtain the stability and instability of the null solution. This is a unique homogeneous stationary solution dependent to the surface diffusion coefficient and roughening control coefficient.

We also illustrated when the surface diffusion stronger than the roughening, the null solution is globally stable. In the meantime, when the roughening is stronger than the surface diffusion, the null solution becomes unstable.

We made many numerical simulations to find that, when the null solution is unstable, non- null stationary solutions have a certain number of ridges in any columns. When the roughening becomes stronger, the numbers of ridges in a column increase.

\section{Acknowledgements}

We appreciate member of the Complex Systems Laboratory of Al-Zahra University for their cooperation. This work has been supported by the Center of International Scientific Studies \& Collaboration (CISSC).

\section{References}

[1] M. D. Johnson, C. Orme, A. W. Hunt, D. Graff,J. Sudijono, L. M. Sauder, and B. G. Orr, Stable and unstable growth in molecular beam epitaxy, Phys. Rev. Lett. 72(1994), 116-119.

[2] S.Azizi and A.Yagi, Dynamical system for epitaxial growth model under Dirichlet conditions, sci. Math. Jpn. (2015), Number 28.

[3] S. Azizi, G. M. Mola and A. Yagi, longtime convergence for epitaxial growth model under Dirichlet conditions, Osaka Journal of Mathematics, (2017), Volume 54, Number 4.

[4] S.Azizi and A.Yagi, Homogeneous stationary solution to epitaxial growth model under Dirichlet conditions. Sci. Math. Japonica. (2018), Number 28.

[5] H. Fujimura and A. Yagi, Dynamical system for BCF model describing crystal surface growth, Vestnik Chelyabinsk Univ. Ser. 3 Mat. Mekh. Inform. 10 (2008), Number 75-88.

[6] H. Fujimura and A. Yagi, Asymptotic behavior of solutions for BCF model describing crystal surface growth, Int. Math. Forum 3 (2008), Number 1803- 1812.

[7] H. Fujimura and A. Yagi, Homogeneous stationary solution for BCF model describing crystal surface growth, Sci. Math. Jpn. (2009), Number 295-302. 